\title{
Study of the imaging characteristics of holographic waveguides
}

Sergio Bleda, Cristian Neipp, Jorge Frances, Mariela Alvarez, Andres Marquez, et al.

Sergio Bleda, Cristian Neipp, Jorge Frances, Mariela Alvarez, Andres Marquez, Sergi Gallego, Inmaculada Pascual, Augusto Beléndez, "Study of the imaging characteristics of holographic waveguides," Proc. SPIE 11030, Holography: Advances and Modern Trends VI, 1103013 (23 April 2019); doi: 10.1117/12.2522167

SPIE. Event: SPIE Optics + Optoelectronics, 2019, Prague, Czech Republic 


\title{
Study of the imaging characteristics of holographic waveguides
}

\author{
Sergio Bleda ${ }^{a}$, Cristian Neipp ${ }^{\text {a }}$, Jorge Frances ${ }^{a}$, Mariela Alvarez ${ }^{a}$, Andres Marquez ${ }^{\text {a }}$, Sergi \\ Gallego $^{\mathrm{a}}$, Inmaculada Pascual ${ }^{\mathrm{a}}$, Augusto Belendez ${ }^{\mathrm{a}}$ \\ ${ }^{a}$ Instituto Universitario de Física Aplicada a las Ciencias y las Tecnologías, Universidad de Alicante. \\ Apartado 99, 03080 Alicante, Spain
}

\begin{abstract}
In this work we study and characterize and holographic waveguide system composed of two volume diffraction gratings, which act as optical couplers and a glass substrate that acts as a waveguide. The features such us high diffraction efficiencies and Bragg angle selectivity exhibited by volume diffraction gratings allow the appropriated adjustment of the incident light to be diffracted in the direction of total reflection condition inside the glass substrate with high efficiency. In this work the holographic waveguide is recorded on different photopolymer materials, which optimization is controlled to obtain high spatial frequencies and stable fringes. The effect of the shrinkage of the holograms after recording in the quality of the image obtained from the optical waveguide is also studied. To perform the experiments two kind of photopolymers were studied: a nanoparticle-(thiol-ene) polymer composite, NPC, and a penta/hexa-acrylate based polymer with dispersed nematic liquid crystal molecules, PDLC. The holographic characteristics such as diffraction efficiency or wavelength selectivity obtained with the waveguides recorded on the different photopolymers were also studied and compared.
\end{abstract}

Keywords: Holography, waveguide, diffraction gratings, volume holography, volume diffraction

\section{INTRODUCTION}

The importance of augmented reality on our Society has increased exponentially during last years, and it seems it will continue expanding greatly in next years due to the versatility it offers to integrate effectively in almost any area. Highly associated with Entertainment, augmented reality offers a rich environment for video games, but it has applications in other different areas such as in drone piloting, surgical training, industry, tourism (it allows to improve the experience of the visitors of a museum or city through the integration of visual or sound content), etc.

Augmented Reality devices usually consist of a headset and a display system to show the user the virtual information that is added to the real one. An Optical See-through Display (glasses) is specially interesting for mobile use because it is safer and posses hands-free and high privacy character. The Google Glass were the pioneers in the Market (launched in 2014) and the ones that have traveled the most since they incorporate functions such as camera, calendar, object recognition while driving, text translation and viewing of photos and videos, among other applications. But in recent years new devices have emerged that have allowed virtual reality (VR) experiences to live such as Oculus Rift in 2015 or HTC VIVE and Microsoft Hololens.

In order to manufacture these devices Holographic techniques play an important role, due to the ability of Holographic optical elements to beam shaping light [1]. A typical see-through display can, for instance, be created by recording two holograms in a glass substrate; the first hologram acting as an in-coupler element, and the second hologram acting as an out-coupler element. Light is guided through the glass substrate by total internal reflection. For this particular application photopolymer materials are good candidates to serve as recording materials. This is due to their well known advantages such as low price, self-processing capability and high versatility [2]. For this particular application the photopolymer composition has to be optimized for the specific requirements, such as low shrinkage, optimum refractive index modulation and recording sensitivity, thickness, etc. In this work, we investigate the responses for different spatial frequency and slanted gratings of two different photopolymers for recording such waveguides: a nanoparticle-(thiol-ene) polymer composite dispersed with $\mathrm{SiO} 2$ nanoparticles [3-5] and average functionality higher than two, NPC, and a photopolymer with dispersed liquid crystal molecules [6], with average functionality higher than five, PDLC. The experiments were performed by using two different recording geometries. The first one is similar to the analyzed in [7],

Holography: Advances and Modern Trends VI, edited by Antonio Fimia, Miroslav Hrabovský, John T. Sheridan,

Proc. of SPIE Vol. 11030, 1103013 - @ 2019 SPIE · CCC code: 0277-786X/19/\$18 - doi: 10.1117/12.2522167 
where the spatial frequency is around 1700 lines $/ \mathrm{mm}$ and the tilt angle of the grating vector with the light propagation inside the material is $69^{\circ}$. The other is the use of a lower spatial frequency,1000 lines $/ \mathrm{mm}$, which requires a tilt angle of the grating vector of 60.77 .

\section{THEORETICAL DESCRIPTION}

\subsection{Waveguide design}

In this section the theoretical basis of the waveguide device is explained. The optical waveguide based on two transmission holograms is shown in Figure 1. Light is incident perpendicularly to the couple-in transmission diffraction grating, which redirects light in a direction accomplishing total internal reflection, whereas the couple-out transmission diffraction grating extracts the light out from the waveguide.

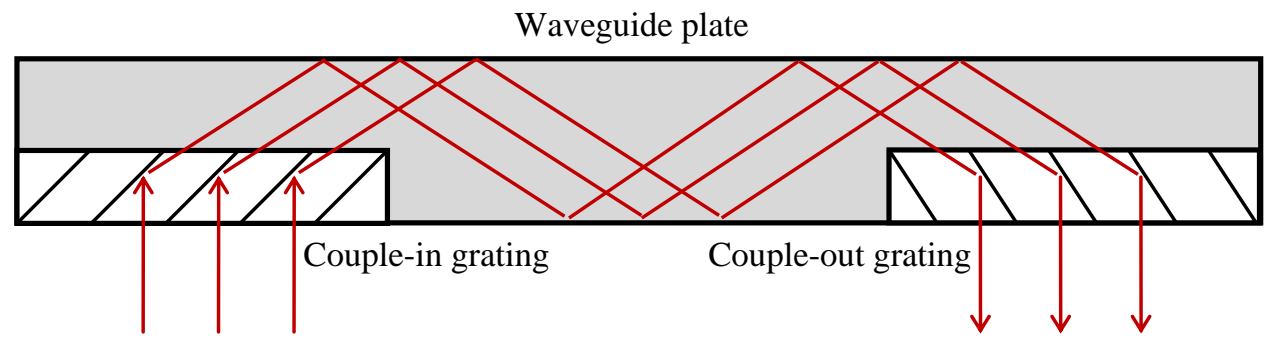

Figure 1. Optical waveguide fabricated with two diffraction gratings acting as in-coupler and out-coupler and glass substrate guiding light by total internal reflection.

\subsection{Reconstruction parameters of the transmission gratings}

The re-direction task of the transmission holographic gratings is performed due to Floquet's condition for gratings, which is explained in Figure 2. In Figure 2 the performance of the couple-in transmission grating can be observed (it should be noticed that the behavior of the couple-out grating is similar to that of the couple-in grating but with light path reversed). $\vec{k}_{i}$ and $\vec{k}_{d}$ are the propagation vectors of the incident and diffracted beam respectively, $\vec{K}$ is the grating vector and $\theta_{g}$ is the critical angle inside the photopolymer. The geometrical parameters of the transmission grating (period, $\Lambda$, and angle of slant of the fringes, $\varphi$ ) can be obtained for a specified wavelength of reconstruction, $\lambda_{c}$, and critical angle inside the grating. The moduli of the propagation vectors are related to the reconstruction wavelength and to the average refractive index of the grating, $n_{g}$, as:

$$
\beta_{c}=\left|\vec{k}_{i}\right|=\left|\vec{k}_{d}\right|=\frac{2 \pi}{\lambda_{c}} n_{g}
$$

The directions of the propagation vectors are prescribed by Figure 3, where $\vec{k}_{i}$ is perpendicular to the grating and $\vec{k}_{d}$ forms an angle $\theta_{g}$ with $\vec{k}_{i}$. Therefore the grating vector $\vec{K}$ can be obtained as:

$$
\vec{K}=\vec{k}_{i}-\vec{k}_{d}
$$

The spatial frequency, $f$, or the period of the grating, $\Lambda$, can be obtained from:

$$
|\vec{K}|=2 \pi f=\frac{2 \pi}{\Lambda}
$$

And the angle $\varphi$ formed by the interference fringes with the substrate can be calculated by using:

$$
\varphi=\operatorname{atan}\left(\frac{K_{y}}{K_{x}}\right)-\frac{\pi}{2}
$$

Where $K_{x}$ and $K_{y}$ are the $\mathrm{x}$ and y components of the $\vec{K}$ vector according to axes criteria of figure 2 . 


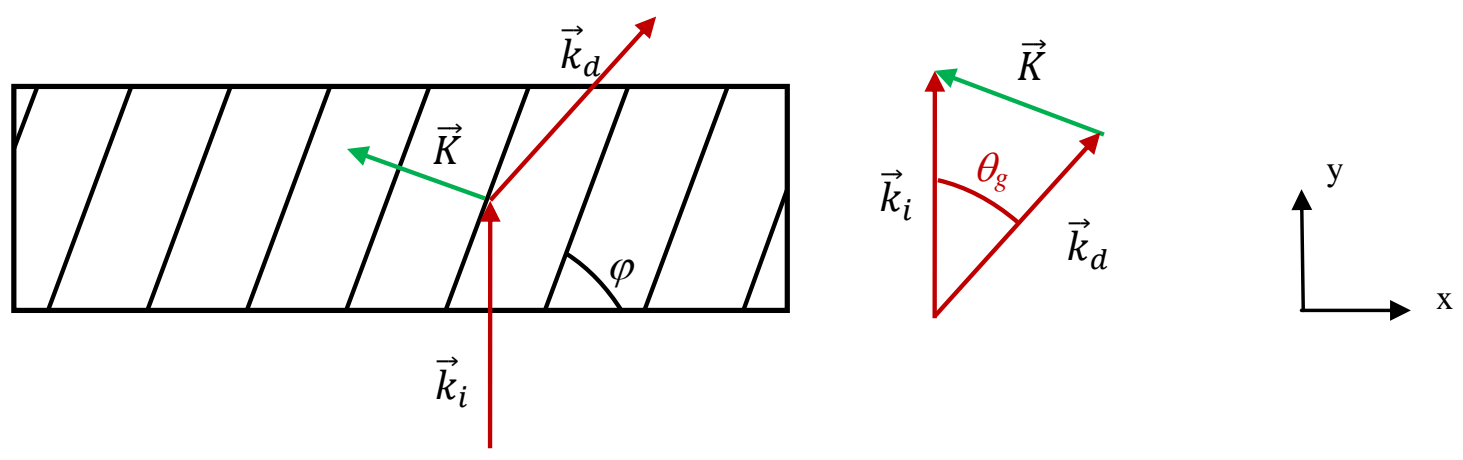

Figure 2. Transmission grating acting as in-coupler element

\subsection{Recording parameters of the transmission gratings}

Although we have found the design parameters of the gratings for the reconstruction process it is clear that these gratings cannot be recorded using the same wavelength as that in the reconstruction step. This is because total internal reflection inside the waveguide is required, so for the recording of a grating with a grating-vector which coupled the incident light into a diffracted wave accomplishing total internal reflection, the reference and object rays should form with the perpendicular to the interface air-polymer an angle of $0^{\circ}$ and $90^{\circ}$, respectively. Therefore the recording of the grating must be made with other wavelength to that of the recording process. Figure 3 illustrates the configuration of Reconstruction and Recording. The propagation vectors $\left(\vec{k}_{r}\right.$, for reference wave, and $\vec{k}_{o}$, for object wave) in the recording process used to obtain the same $\vec{K}$ vector as that of the reconstruction step, but with a different wavelength, are different to those of the recording process. If, $\beta_{r}$, is the modulus of these vector, they are obtained as:

$$
\beta_{c}=\left|\vec{k}_{r}\right|=\left|\vec{k}_{o}\right|=\frac{2 \pi}{\lambda_{r}} n_{p}
$$

Where $\lambda_{r}$ is the recording wavelength and $n_{p}$ is the refractive index of the photopolymer.

It is possible to obtain the propagation vectors, $\vec{k}_{r}$ and $\vec{k}_{o}$, for prescribed values of $\vec{K}$ and $\beta_{r}$ (the radius of Ewald sphere) by using the following geometrical arguments:

Since $\vec{k}_{r}$ and $\vec{k}_{o}$ have the same modulus the vectors $\left(\vec{k}_{r}-\vec{k}_{o}=\vec{K}\right)$ and $\left(\vec{k}_{r}+\vec{k}_{o}\right)$ are perpendicular, so the dot product among them is zero:

On the other hand:

$$
\vec{K} \cdot\left(\vec{k}_{r}+\vec{k}_{o}\right)=0
$$

$$
\vec{K} \cdot \vec{K}=\vec{K} \cdot\left(\vec{k}_{r}-\vec{k}_{o}\right)=|\vec{K}|^{2}
$$

From the two previous expressions the following equation can be obtained:

$$
|\vec{K}|^{2}=2 \vec{K} \cdot \vec{k}_{r}=-2 \vec{K} \cdot \vec{k}_{o}
$$

Therefore the angles of $\vec{k}_{r}$ and $\vec{k}_{o}$ with $\vec{K}$ are determined by:

$$
\begin{gathered}
\cos \left(\theta_{\overrightarrow{\vec{K}_{k}}}\right)=\frac{|\vec{K}|}{2\left|\vec{k}_{r}\right|} \\
\cos \left(\theta_{\overrightarrow{\vec{K}^{*}} \vec{k}_{o}}\right)=-\frac{|\vec{K}|}{2\left|\vec{k}_{o}\right|}
\end{gathered}
$$

Since the modulus of $\vec{k}_{r}$ and $\vec{k}_{o}$ are prescribed $\left(\beta_{r}\right)$, these vectors are completely determined. 

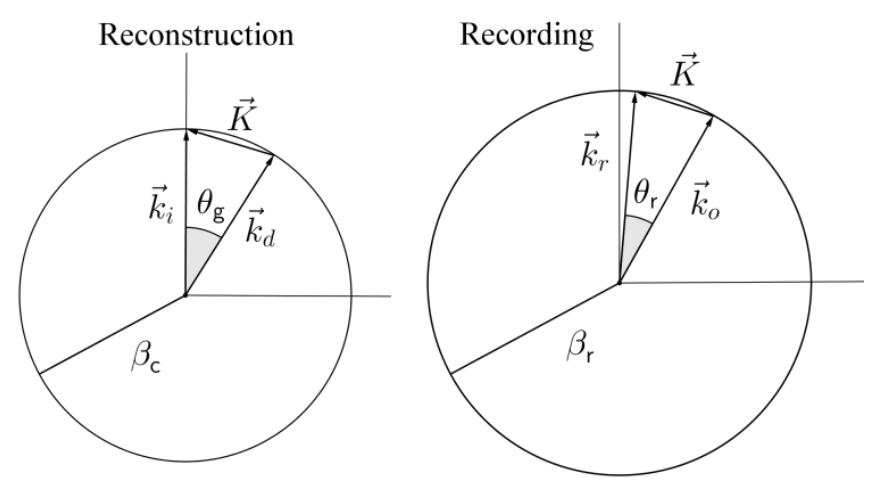

Figure 3. Recording and reconstruction parameters for a transmission diffraction grating

\section{EXPERIMENTAL SET-UP}

In order to perform the experiments the experimental set-up of figure 4 was used. Changing the angle of the sample holder from this initial configuration, slanted gratings with different geometries can be obtained. The intensity of the initial beam was of $2.4 \mathrm{~mW} / \mathrm{cm}^{2}$; and this beam was split in two beams with different intensities so as to achieve a beam ratio near 1:1 in the interference zone on the material.

Two different polymer compositions were used in the experiments:

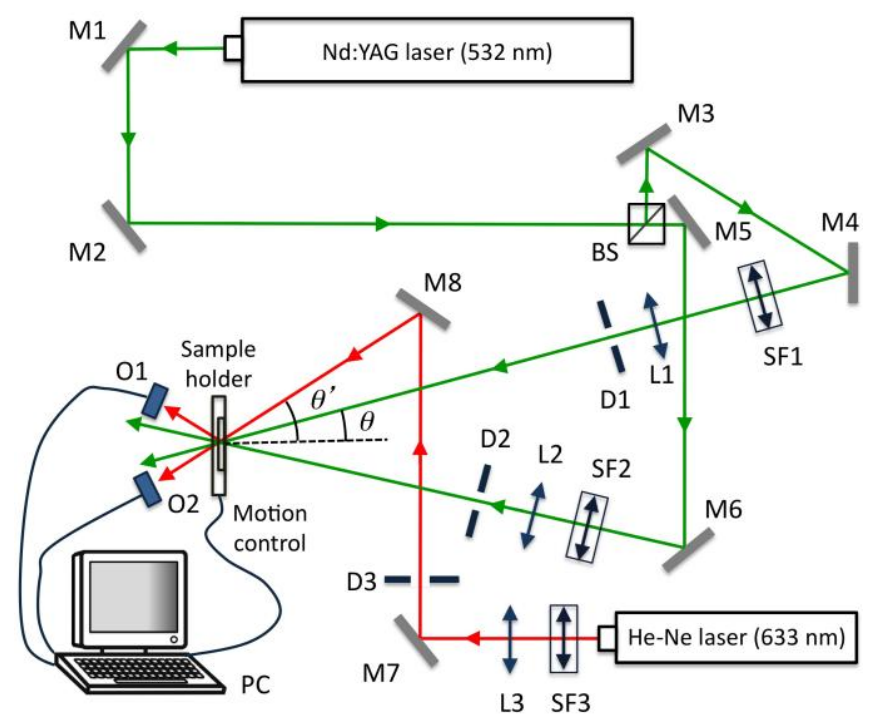

Figure 4. Experimental setup.

The first composition was a thiol-ene based NPC system. This composition consisted of the stoichiometric mixture of thiol-ene monomer, containing allyl triazine ene monomer, triallyl-1,3,5-triazine-2,4,6(1H,3H,5H)-trione (Aldrich), and dithiol monomer, 1,4-bis(3-mercaptobutyryloxy) butane (Aldrich). SiO2 nanoparticles (with average size of $13 \mathrm{~nm}$ ), were dispersed in the thiol-ene monomer blend [8]. With this mixture large values of the refractive index can be obtained, due to the fact that the refractive index difference between $\mathrm{SiO} 2$ nanoparticles and the formed thiol-ene polymer is close to 0.1 . Another effect of the the dispersed nanoparticles is to improve the thermal stability of the gratings recorded in the NPC material [5]. The efficient polymerization of the thiol-ene NPC material by a green laser was achieved by the addition of 2 wt. \% of titanocene organo-metallic complex (Chivacure 534, Chitec Tech.) in combination with 2.5 wt. \% benzoyl peroxide (BzO2, Aldrich). The mixed syrup was dripped on a glass substrate and 
dried in an oven at $55^{\circ} \mathrm{C}$ for 20 minutes to eliminate the MIBK solvent. Then, another glass substrate was placed to cover the dipped syrup. We used 20-30 $\mu \mathrm{m}$ glass microspheres as spacers between the two glass substrates.

The other material analyzed is PDLC. The monomer used was dipentaerythritol penta-/hexaacrylate (DPHPA), with a refractive index of $n=1.490$. For the liquid crystal we used the nematic liquid crystal BL036 from Merck, which has an ordinary refractive index $n_{0}=1.5270$ and a difference between extraordinary and ordinary index $\Delta n=0.2670$ [9]. As a crosslinker we used N-vinyl-2-pyrrolidone (NVP), as radical generator N-phenylglicine (NPG), octanoic acid (OA) as cosolvent [9] and ethyl eosin (YEt) as dye. N-methyl-2- pyrrolidone was used in combination with NVP in order to control overmodulation during hologram recording [9].

\section{RESULTS AND DISCUSSION}

Experiments were conducted by using two different materials and also two different geometries. Firstly, slanted gratings were recorded in thiol-ene based NPC material. The recording wavelength was in this case of $532 \mathrm{~nm}$ in the recording step and $633 \mathrm{~nm}$ in the reconstruction step. The refractive index of the glass substrate (acting as the waveguide) was of $\mathrm{n}=1.51$, which gives a value of $41.47^{\circ}$ for the critical angle of total internal reflection. For the gratings, the refractive index was taking as $n_{g}=1.49$, thus giving a critical angle of 42.2 inside the grating. The angles made by the reference and object beam in air with respect to the normal to the interface air-photopolymer were $26^{\circ}$ and $73^{\circ}$, respectively. The resulting grating with this configuration had a spatial frequency $\sim 1000$ lines $/ \mathrm{mm}$ and a slant angle (angle formed by the $\vec{K}$ vector with the y axis (figure)) of $60.77^{\circ}$. In this case the incident red beam (in the reconstruction step) formed an angle of $\sim 23^{\circ}$ in air with respect to the normal to the interface air-grating to achieve total internal reflection. Figure 5 shows the angular response of the transmission efficiency for a slanted transmission grating recorded in the NPC material with 1000 lines $/ \mathrm{mm}$. Obtaining experimental data of the transmission efficiency instead of diffraction efficiency as a function of the angle is due to the fact that the diffracted beam is trapped inside the glass substrate at a diffraction angle larger than the critical angle at the boundary air-glass substrate and the air. The experimental data were fitted to the theoretical curve using Kogelnik's coupled wave theory [10], providing a value of the thickness of $d=19 \mu \mathrm{m}$ and a refractive index modulation of $n_{l}=0.011$. It can be observed from the figure that the transmission efficiency is only of $20 \%$, so the main percentage of light is guided into the glass substrate.

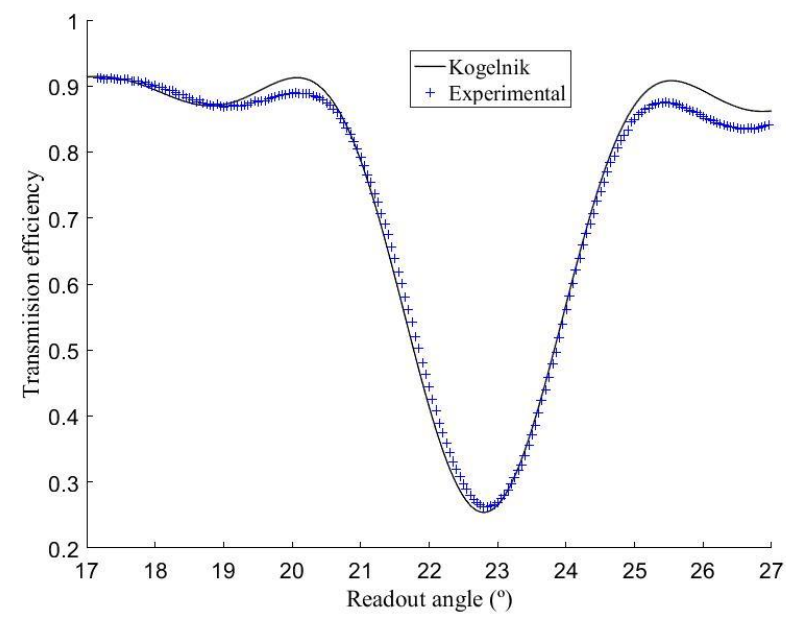

Figure 5. Experimental and theoretical angular response of the transmission efficiency for a 1000 lines/mm transmission diffraction efficiency recorded on NPC material

The other material used in the experiments was the PDLC photopolymer, which has demonstrated good behavior for symmetric gratings with spatial frequencies around 1000 lines $/ \mathrm{mm}$ for different thicknesses [11], and a functionality between 5 and 6 . The main interest in this kind of material is that it confers the material switchable characteristics. The angles made by the reference and object beam in air with respect to the normal to the interface air-photopolymer were $5.2^{\circ}$ and $68.5^{\circ}$, respectively. The resulting grating with this configuration had a spatial frequency $\sim 1700$ lines $/ \mathrm{mm}$ and a 
slant angle (angle formed by the $\vec{K}$ vector with the y axis (figure)) of $68.9^{\circ}$. In this case the incident red beam (in the reconstruction step) was incident perpendicularly to the normal to the interface air-grating to achieve total internal reflection. Figure 6 shows the angular response of the transmission efficiency for a slanted transmission grating recorded on the PDLC material with 1700 lines $/ \mathrm{mm}$. The fitting values in this case were: a thickness of $d=47 \mu \mathrm{m}$ and a refractive index modulation of $n_{l}=0.0038$. It can be observed from the figure that the transmission efficiency is less than $10 \%$, so more than the $90 \%$ of light is guided into the glass substrate in this case. The width of the central lobe is lower than that in the NPC case, since the thickness of the grating is higher in this case. Therefore for PDLC materials higher angular (and wavelength) selectivity is acquired with respect to the NPC material. The comparison of figures 6 and 7 also shows that whereas in the gratings recorded on the NPC material the transmittance curves present a symmetrical behavior around the central lobe, this is not the case for the gratings recorded on the PDLC material. This suggests that shrinkage is more important for the PDLC material which is in agreement with previous studies reporting substantive shrinkage suppression as a result of $\mathrm{SiO} 2$ dispersion in thiol-ene NPC $[4,5]$.

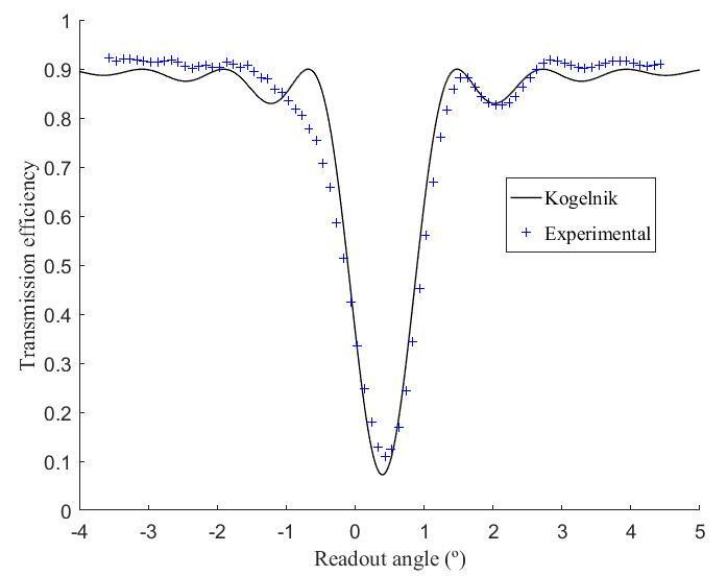

Figure 6. Experimental and theoretical angular response of the transmission efficiency for a 1000 lines/mm transmission diffraction efficiency recorded on PDLC material

We finally present in Figure 7 a holographic waveguide fabricated with the non-slanted gratings of 1700 lines $/ \mathrm{mm}$ previously analized, where it is visible that the holographic waveguide guides light inside the glass substrate from the incouple to the out-couple transmission holographic gratings.

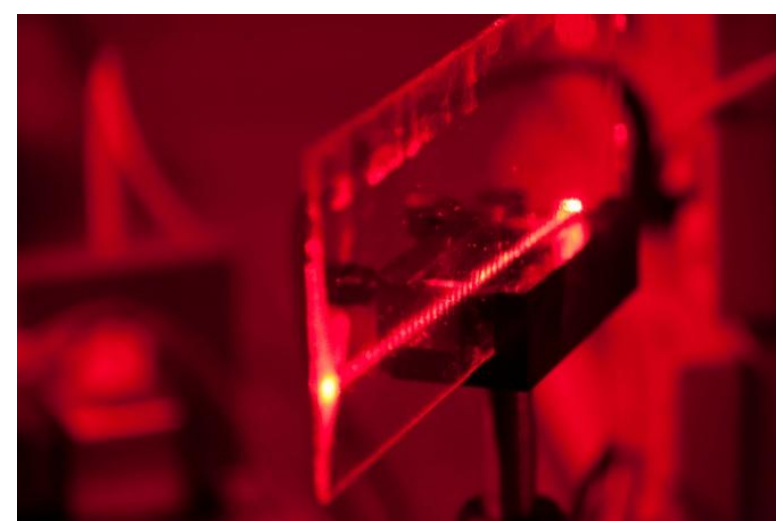

Figure 7. Holographic waveguide 


\section{CONCLUSIONS}

The fabrication of waveguides in two different photopolymers using two different geometries has been evaluated in this work. The holographic waveguide consists of two transmission holographic gratings acting as in-coupler and out-coupler and the glass substrate acting as the waveguide. The theoretical framework for this kind of design has been presented showing that in order to record the transmission gratings a different wavelength in the recording to that of the reconstruction must be used so as to guide the light by total internal reflection. A scheme to calculate the recording parameters knowing the reconstruction ones has been presented. For the recording of the waveguides two polymers have been used: a thiol-ene based NPC with the monomer functionality between 2 and 3 and a PDLC material with functionality higher than 5 . A better behavior with respect to shrinkage was obtained using the NPC material. In both cases a high percentage of the incident light is guided through the substrate.

\section{ACKNOWLEDGMENTS}

This work was supported by the "Ministerio de Economía Industria y Competitividad" (Spain) under projects FIS201782919-R (MINECO/AEI/FEDER, UE) and FIS2015-66570-P (MINECO/FEDER). and by "Generalitat Valenciana" (Spain) (PROMETEOII/2015/015). R. F. and Y. T., acknowledge a financial support by the Ministry of Education, Culture, Sports, Science and Technology of Japan under grant $15 \mathrm{H} 03576$.

\section{REFERENCES}

[1] Zhang, N., Liu, J., Han, J., Li, X., Yang, F., Wang, X., Hu. B. and Wang, Y., “Improved holographic waveguide display system," Appl. Opt. 54, 3645-3649 (2015).

[2] Guo, J., Gleeson, M. R. and Sheridan, J. T., "A Review of the Optimisation of Photopolymer Materials for Holographic Data Storage,” Phys Res. Int., Article ID 803439, 16 pages, (2012).

[3] Hata, E. and Tomita, Y., "Order-of-magnitude polymerization-shrinkage suppression of volume gratings recorded in nanoparticle-polymer composites," Opt. Lett. 35, 396-398 (2010).

[4] Hata, E., Mitsube, K., Momose, K., Tomita, Y., "Holographic nanoparticle-polymer composites based on stepgrowth thiol-ene photopolymerization," Opt. Mat. Express 1, 207-222 (2011).

[5] Hata. E. and Tomita, Y., "Stoichiometric thiol-to-ene ratio dependences of refractive index modulation and shrinkage of volume gratings recorded in photopolymerizable nanoparticle-polymer composites based onstepgrowth polymerization," Opt. Mater. Express 1, 1113-1120 (2011).

[6] Ortuño, M., Riquelme, M., Gallego, S., Márquez, A., Pascual, I. and Beléndez, A., "Overmodulation Control in the Optimization of a H-PDLC Device with Ethyl Eosin as Dye," Int. J. Polym. Sci., Article ID 357963, 8 pages (2013).

[7] Neipp, C., Francés, J., Martínez, F. J., Fernández, R., Alvarez, M.L., Bleda, S., Ortuño, M., Gallego ,S., "Optimization of Photopolymer Materials for the Fabrication of a Holographic Waveguide," Polym. 9(9), 395 (2017).

[8] Fernández, R., Gallego, S., Navarro-Fuster, V., Neipp, C., Francés, J., Fenoll, S., Pascual, I. and Beléndez, A., "Dimensional changes in slanted diffraction gratings recorded in photopolymers," Opt. Mater. Express 6, 34553468 (2016).

[9] Ortuño, M., Riquelme, M., Gallego, S., Márquez, A., Pascual, I. and Beléndez, A., "Overmodulation Control in the Optimization of a H-PDLC Device with Ethyl Eosin as Dye," Int. J. Polym. Sci., Article ID 357963, 8 pages, (2013).

[10] Kogelnik, H., "Coupled wave theory for thick hologram gratings". Bell Labs Tech. J. 48, 2909-2947 (1969).

[11] Gallego, S., Ortuño, M., Márquez, A., Fernández, R., Álvarez, M., Pascual, I. and Beléndez, A., "Influence of Thickness on the Holographic Parameters of H-PDLC Materials,” Int. J. Polym. Sci., 528287 (2014). 